\title{
Progressive diffuse large B-cell lymphoma with TP53 gene mutation treated with chidamide-based chemotherapy
}

\author{
Qing $\mathrm{Li}^{1}$, Jingcao Huang ${ }^{1}$, Yang $\mathrm{Ou}^{1}$, Yan $\mathrm{Li}^{1} \&$ Yu Wu${ }^{*, 1}$ \\ ${ }^{1}$ Department of Hematology \& Hematology Research Laboratory, West China Hospital, Sichuan University, Chengdu, Sichuan \\ 610041, PR China \\ *Author for correspondence: Tel.: +86 288542 2370; Fax: +86 288542 3921; wu_yu@scu.edu
}

We attempted to explore novel treatment options for progressive diffuse large B-cell lymphoma (DLBCL) with TP53 mutation that has a poor response to rituximab-based immunochemotherapy. Herein, we report the case of a patient with DLBCL having TP53 mutation who showed progression following four cycles of rituximab-based immunochemotherapy but achieved sustained partial remission following chidamidebased chemotherapy. In vitro experiments performed using the DLBCL cell lines OCI-ly1 (LY1; mutant TP53), OCl-ly10 (LY10; wild-type TP53) and OCI-ly19 (LY19, wild-type TP53) demonstrated that chidamide is more potent against cells with mutant TP53 mutant than those with wild-type TP53. Moreover, chidamide can reduce the mRNA and protein expression levels of mutant TP53 and upregulate the surface expression of the CD20 antigen in lymphoma cells.

First draft submitted: 19 June 2018; Accepted for publication: 4 December 2018; Published online: 4 January 2019

Keywords: CD20 • chidamide • diffuse large B-cell lymphoma • immunochemotherapy • immunomodulator • thalidomide $\bullet$ TP53 mutation

\section{Presentation \& initial diagnosis}

The patient was a 74-year-old man who was diagnosed with diffuse large B-cell lymphoma (DLBCL) nongerminal center subtype (immunohistochemistry) stage III using lymph node biopsy. Immunohistochemical analysis of tumor cells indicated positivity for CD20, BCL-6 and MUM-1. The positivity of Ki-67/MIB, C-MYC and BCL-2 were 70, 20 and 80\%, respectively. Tumor cells were negative for CD3, CD10, CD30, GB, TIA-1/ALK-1 and EMA (Figure 1). The fluorescence in situ hybridization performed suggested that the $B C L-2$ gene copy number was increased, but there were no $B C L-2, B C L-6$ or $C-M Y C$ gene translocations. Additionally, PET-CT conducted revealed multiple lymphadenopathies in the neck, abdomen and spleen areas (Figure 2).

\section{Treatment}

The patient was treated using R-CHOP (rituximab $375 \mathrm{mg} / \mathrm{m}^{2}$ intravenous infusion [iv.], D1; cyclophosphamide $750 \mathrm{mg} / \mathrm{m}^{2}$ [iv.], D2; vincristine $1.4 \mathrm{mg} / \mathrm{m}^{2}$ [iv.], D2; doxorubicin hydrochloride $50 \mathrm{mg} / \mathrm{m}^{2}$ [iv.], D2; and prednisone $50 \mathrm{mg}$ p.o. b.i.d., D2, 3, 4, 5 and 6). He experienced several pulmonary infections during postchemotherapy myelosuppression. Following four ccles of immunochemotherapy, the lymph nodes re-enlarged, and he experienced abdominal pain. PET-CT revealed newly emerging lymph nodes in the neck and abdomen (Deauville score, 5 points) and enlargement of the para-aortic lymph nodes with increased glucose metabolism (Figure 2). Considering the progressing disease and the vulnerable status of the patient, ctDNA technology (sequencing platform, Illumina; detection method, NGS; Wuhan Baitai Medical Laboratory, Wuhan, China; coverage depth, up to $4218 \times$ ) was used as previously described by Alizadeh [1] and Rossi [2], it detected a TP53 gene mutation at site P.R248Q, with a variant allele fraction of $1.3 \%$. Chidamide-based chemotherapy was administered. The specific chemotherapy regimen included rituximab $375 \mathrm{mg} / \mathrm{m}^{2}$ iv. monthly, chidamide $30 \mathrm{mg}$ p.o. b.i.w., prednisone $20 \mathrm{mg}$ p.o. q.o.d., cyclophosphamide $50 \mathrm{mg}$ p.o. q.o.d. and thalidomide $50 \mathrm{mg}$ p.o. q.n. After 2 months of chidamide-based chemotherapy, the patient's lymph nodes unexpectedly shrank to an inaccessible size, and the regimen was continued. PET-CT was performed 3 months later to evaluate the response to the novel regimen. Except for stable 


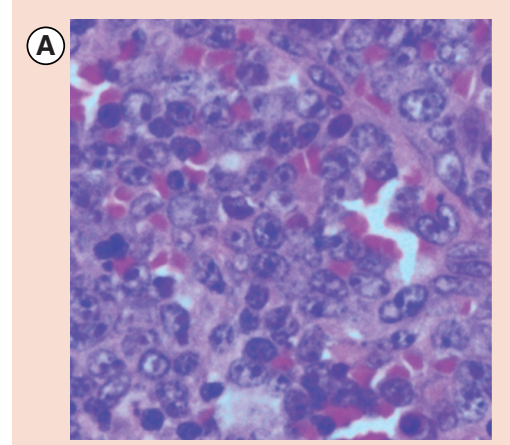

(D)

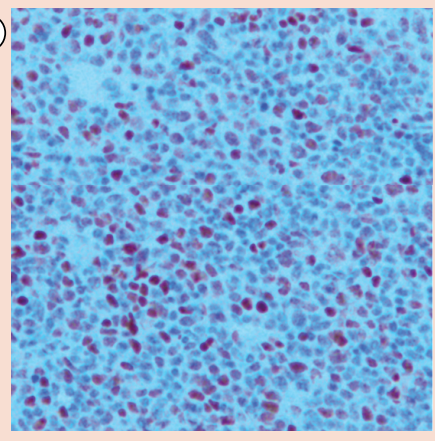

(B)

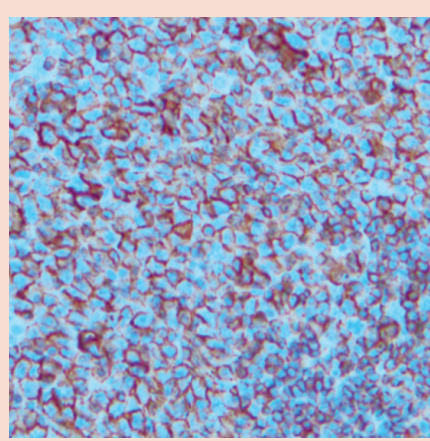

(E)

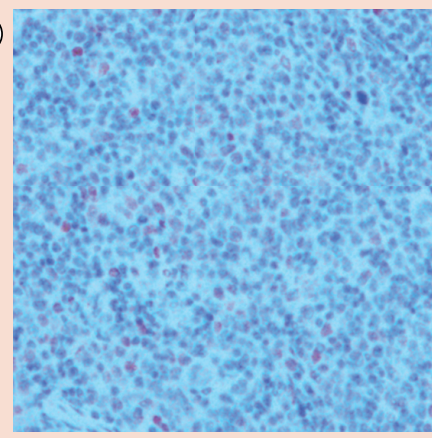

(C)

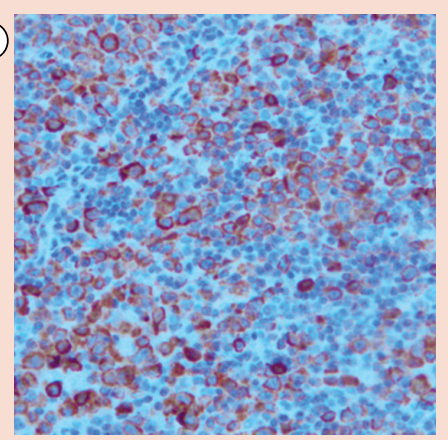

(F):

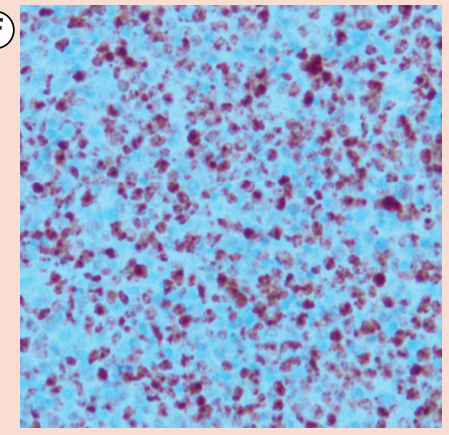

Figure 1. Lymph node biopsy and immunohistochemical staining. (A) Lymph node biopsy (hematoxylin and eosin, $\times 400)$; (B) positive for CD20; (C) positive for BCL-2 (+, 80\%); (D) positive for BCL-6; (E) positive for C-MYC (+, $20 \%)$; (F) positive for P53 $(+,>90 \%)(B-F, \times 200)$.

findings in lymph nodes in the right cervical region, the glucose metabolism levels in the remaining lymph nodes showed reductions, and no lesions were observed in the left cervical or para-aortic lymph nodes (Figure 2).

\section{Outcome}

The patient has survived for $>1$ year since the date of diagnosis. He is in a good condition with no new lesions, and partial remission has been maintained for up to 9 months. Adverse reactions included mild fatigue and gastrointestinal discomfort among others.

\section{In vitro experiments}

We performed in vitro experiments to determine whether chidamide is more potent against cells with mutant TP53 than those with wild-type TP53.

\section{Methods}

\section{Cell lines}

The transformed DLBCL cell lines OCI-ly1 (LY1) (mutTP53), OCI-ly10 (LY10) (wtTP53) [3] and OCIly19 (LY19) (wt TP53) [4] were obtained from the State Key Laboratory of Biotherapy, Sichuan University, Sichuan, China.

Written informed consent was obtained from the patient, and the study protocol was approved by the Ethics Committee. The study was performed in accordance with the Declaration of Helsinki.

\section{Cell viability assay}

Cell viability was determined using an MTT proliferation assay kit (Biosharp Life Sciences, Anhui, China), according to the manufacturer's instructions. Chidamide was tested at concentrations from 0.1 to $25 \mu \mathrm{mol} / \mathrm{l}$ to determine growth inhibition at 48 or $72 \mathrm{~h}$ after treatment in all cell lines. The concentration of the drug that inhibited $50 \%$ of cells $\left(\mathrm{IC}_{50}\right)$ was calculated. The experiments were repeated twice, and each sample had three duplications. 

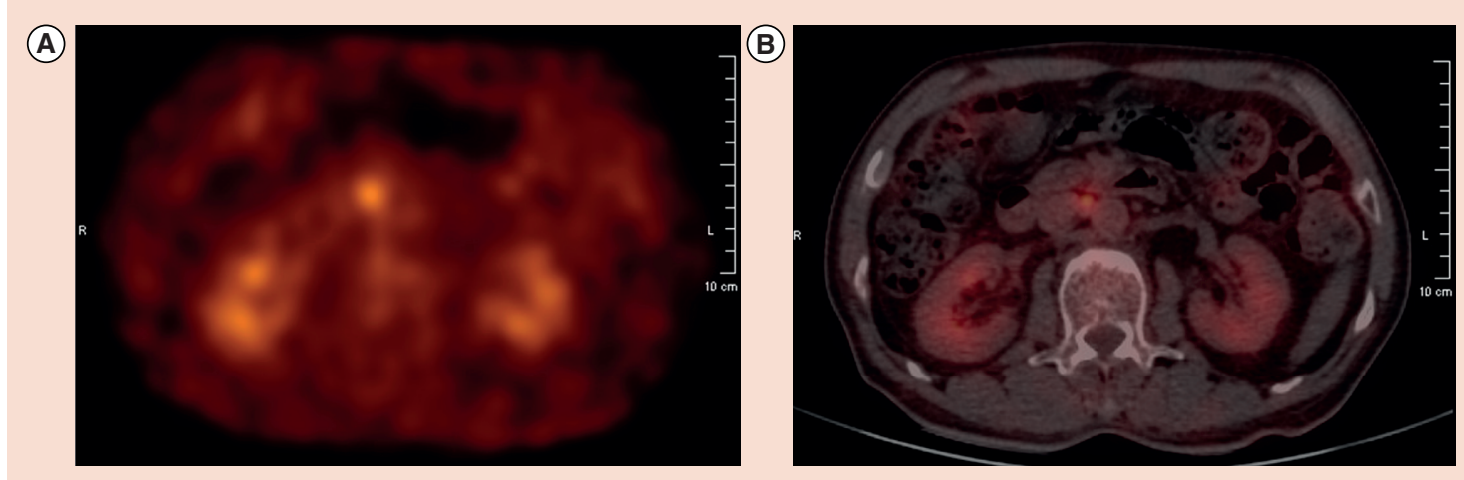

(C)
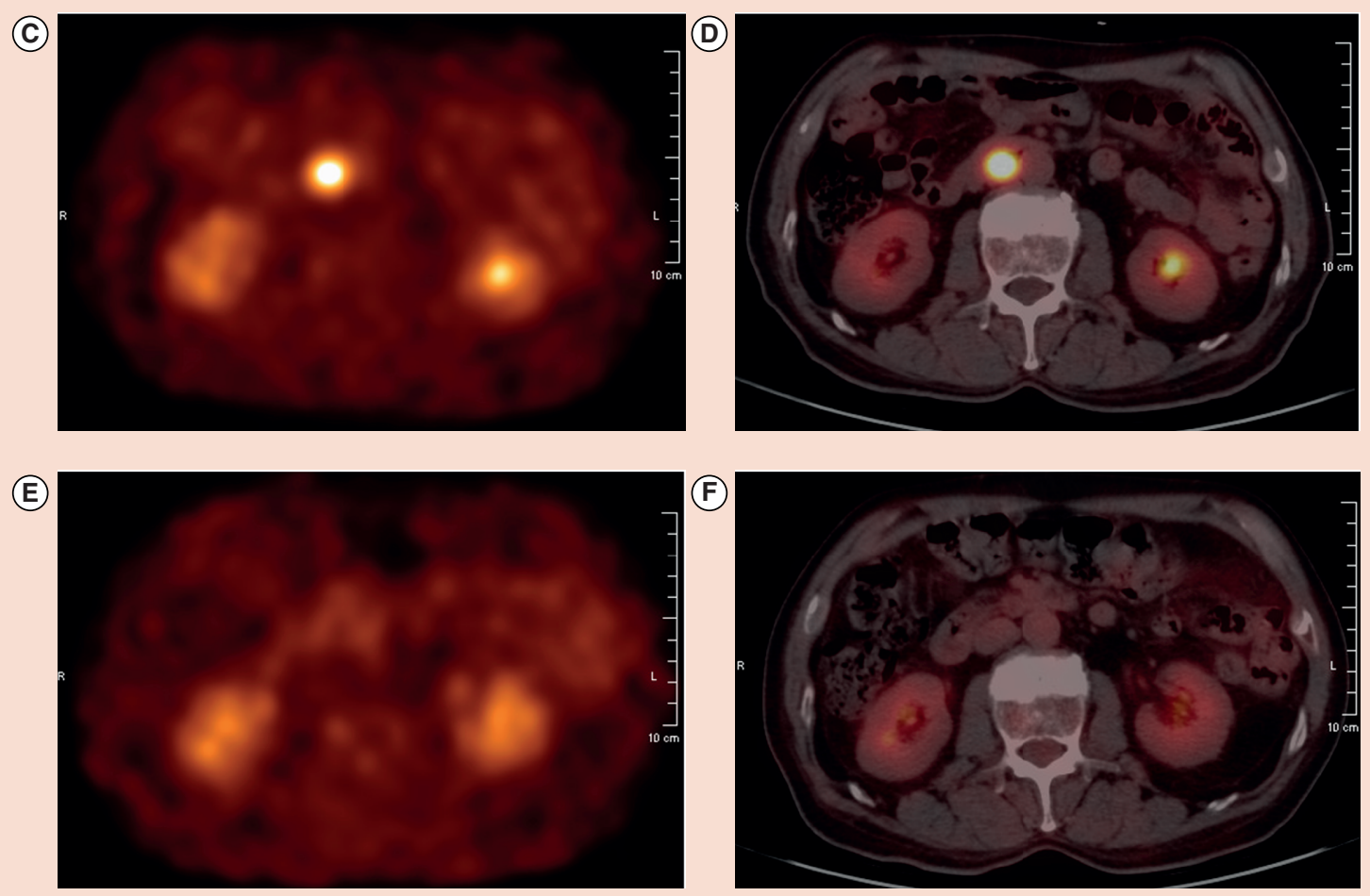

Figure 2. PET-CT results of the patient at the initial diagnosis, during disease progression and at the final follow-up evaluation following chidamide-based chemotherapy. (A \& B) PET-CT image of the patient at the initial diagnosis shows lymphoma invasion. (C \& D) Following four cycles of rituximab-based immunochemotherapy, additional PET-CT shows that the para-aortic lymph nodes are larger than before, with a higher level of glucose metabolism, indicating disease progression. (E \& F) After 4 months of a chidamide-based chemotherapy regimen, another PET-CT shows no lesions in the para-aortic lymph nodes. Overall, the patient shows sustained partial remission.

\section{Real-time polymerase chain reaction assay}

Total RNA was extracted from cell lines using Trizol reagent (MRC, OH, USA), according to the manufacturer's instruction. The expression of target genes was analyzed with real-time quantitative polymerase chain reaction using the SYBR green real-time PCR system (Promega, Shanghai, China). The expression of the housekeeping gene GAPDH was used as an internal control. The primers used for TP53 were 5'-ACCTATGGAAACTACTTCCTGAAAA$3^{\prime}$ and 5'-CAATATCGTCCGGGGACAGC-3' separately. The primers used for GAPDH were 5'CGCTGAGTACGTCGTGGAGTC-3' and 5'-GCTGATGATCTTGAGGCTGTTGTC-3' separately. 

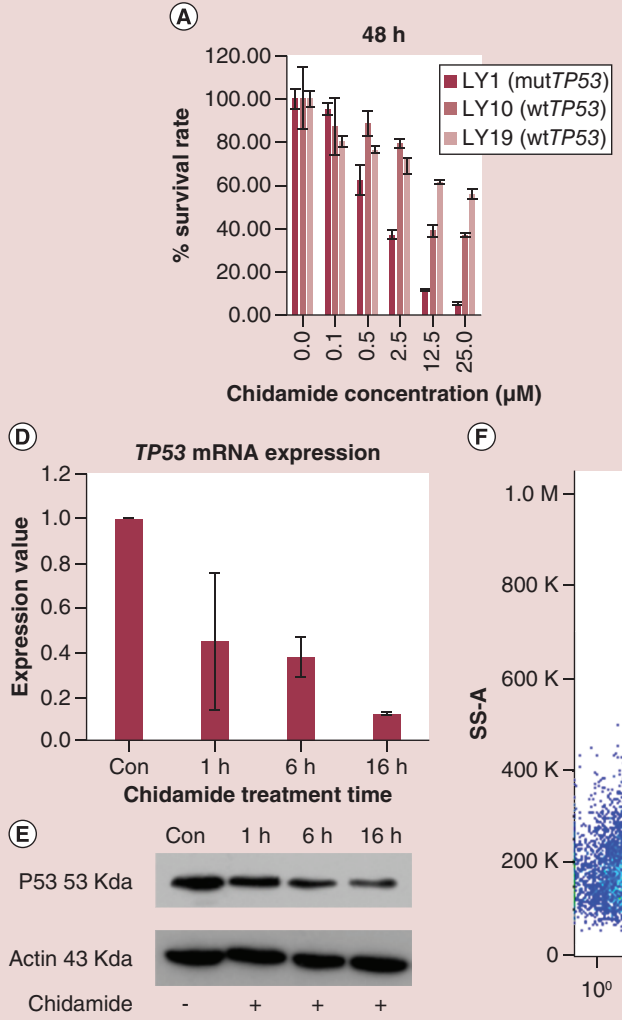

Chidamide
(F)

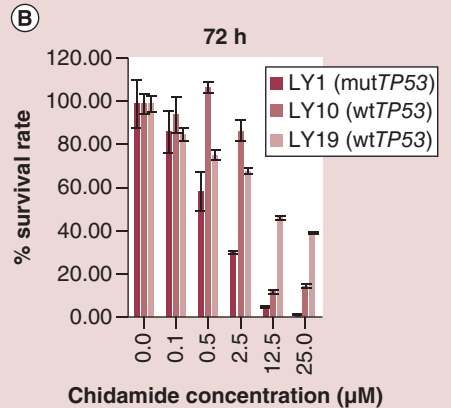

Chidamide concentration $(\mu \mathrm{M})$

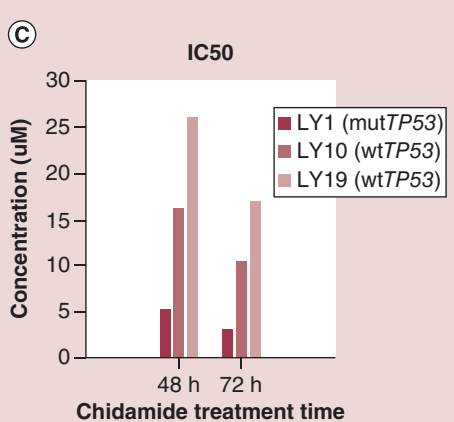

(G)

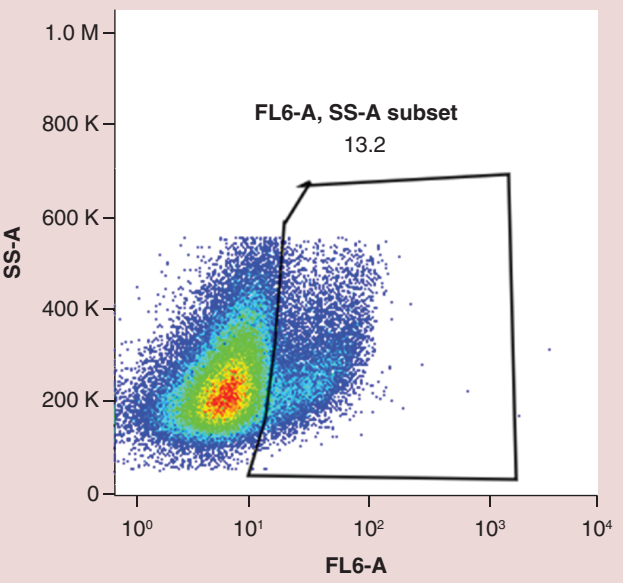

Figure 3. In vitro experiments involving chidamide treatment in OCI-ly1 (LY1) (mutTP53), OCI-ly10 (LY10) (wtTP53) and OCI-ly19 (LY19) (wtTP53) lymphoma cell lines. (A \& B) On chidamide treatment in the three lymphoma cell lines for 48 and $72 \mathrm{~h}$, the survival rate of LY1 (mutTP53) cells is lower than the rates of LY10 (wtTP53) and LY19 (wtTP53) cells ( $<<0.05)$. (C) Following chidamide treatment in the three lymphoma cell lines for 48 and $72 \mathrm{~h}$, the half-maximal inhibitory concentration $\left(I_{50}\right)$, calculated using SPSS software, of LY1 (mutTP53) cells is lower than the values of LY10 (wtTP53) and LY19 (wtTP53) cells ( $p<0.05)$. (D) LY1 (mutTP53) cells are treated with $5.0 \mu \mathrm{M}$ chidamide $\left(\mathrm{IC}_{50}, 48 \mathrm{~h}\right)$ for 1,6 and $16 \mathrm{~h}$, and the cells are then harvested for real-time quantitative polymerase chain reaction analysis. The expression of TP53 mRNA shows a decrease with the extension of the chidamide treatment time. (E) LY1 (mutTP53) cells are treated with chidamide for 1,6 and $16 \mathrm{~h}$ before collection and protein extraction. Western blot analysis performed reveals that chidamide reduces the protein expression of mutant TP53. (F \& G) The expression of the CD20 in LY1 (mutTP53) cells treated with chidamide $(5.0 \mu \mathrm{M})$ for $72 \mathrm{~h}$ is compared with that in control cells using flow cytometry. (F) The expression rate of the CD20 in LY1 (mutTP53) cells in the control group is $1.63 \%$. (G) The expression rate of the CD20 in LY1 (mutTP53) cells in the treatment group is $13.2 \%$.

\section{Western blot analysis}

Western blot analysis was performed according to a standard method [5] using the anti-P53 antibody (Santa Cruz Biotechnology, CA, USA) and anti- $\beta$-actin antibody (4A Biotech, Beijing, China). Finally, reactive proteins were visualized using a chemiluminescence kit (Millipore, MA, USA).

\section{Flow cytometry analysis}

Following drug treatment, the cells were processed according to a standard method, which involved incubation with anti-CD20 antibody (BD, Pharmingen, Shanghai, China), and the results were analyzed using a Navious flow cytometer (Beckman Coulter, CA, USA).

\section{Results}

Chidamide is more potent against DLBCL cells with mutant TP53 than those with wild-type TP53 The survival rate of LY1 (mutTP53) cells was lower than the rates of LY10 (wtTP53) and LY19 (wtTP53) cells following drug treatment for 48 or $72 \mathrm{~h}$ (Figure $3 \mathrm{~A} \& \mathrm{~B}$ ); the differences were statistically significant $(\mathrm{p}<0.0001)$. On treating the cell lines with chidamide concentrations of 12.5 and $25.0 \mu \mathrm{M}$ for $48 \mathrm{~h}$, the survival rates were 11.84 and 5.51\% for LY1 (mutTP53) cells, 39.14 and 37.04\% for LY10 (wtTP53) cells and 61.41 and 56.16\% for LY19 (wt TP53) cells, respectively. The $\mathrm{IC}_{50}$ was calculated using SPSS Statistics software (IBM, NY, USA). 
After $72 \mathrm{~h}$ of treatment, the $\mathrm{IC}_{50}$ of LY1 (mutTP53) cells $(3.007 \mu \mathrm{M}$ ) was lower than the values of LY10 (wt TP53) $(10.452 \mu \mathrm{M})$ and LY19 (wt TP53) cells $(16.916 \mu \mathrm{M})$ (Figure 3C).

\section{Chidamide inhibited the transcription \& translation of mutated TP53}

LY1 (mutTP53) cells were treated with chidamide $5.0 \mu \mathrm{M}$ ( $\mathrm{IC}_{50}$ value at $48 \mathrm{~h}$ ) for 1,6 and $16 \mathrm{~h}$. The cells were collected at various time points to extract RNA and protein. Real-time quantitative polymerase chain reaction and western blot analysis showed that the inhibition of TP53 was time-dependent both at the mRNA and protein levels (Figure 3D \& E).

\section{Chidamide upregulated the surface expression of the CD20 antigen in lymphoma cells}

Following treatment with chidamide $(5 \mu \mathrm{M})$ for $72 \mathrm{~h}$, the expression rate of the CD20 on LY1 (mutTP53) cells was $13.2 \%$, whereas the expression rate on untreated cells was only $1.63 \%$ (Figure $3 F \& G$ ).

\section{Discussion}

DLBCL is the most common non-Hodgkin's lymphoma worldwide and is biologically aggressive [6]. TP53 mutations are the most common mutations in DLBCL and account for $20-25 \%$ of cases $[7,8]$. The prognostic significance of TP53 mutations in patients with DLBCL has been reported in many studies [9]. A TP53 mutation is often investigated in patients with R-CHOP failure and is considered to be an adverse factor in DLBCL [10]. A previous study has reported that the rates of complete remission, overall survival and survival at 5 years were significantly lower in patients with tumors having TP53 mutations than in those with tumors having the wild-type TP53 [11]. Here, we reported a case of patient with DLBCL having TP53 mutation that was resistant to R-CHOP. Partial durable relief was obtained using a 2-month chidamide-based regimen. Moreover, the in vitro experiments demonstrated that chidamide has a greater lethal effect on DLBCL cell lines with mutant TP53 than on cell lines with wild-type TP53. Furthermore, chidamide has a therapeutic effect on DLBCL and may act by inhibiting the transcription and translation of mutated TP53 and upregulating the surface expression of the CD20 antigen in lymphoma cells. To the best of our knowledge, this is the first reported case of refractory DLBCL showing a durable response to chidamide treatment.

Currently, the standard chemotherapy regimen for CD20-positive DLBCL is R-CHOP. Despite the invasive nature of this disease, $50-70 \%$ of patients can be cured using this regimen [12]. However, the R-CHOP regimen is not suitable for the remaining $30-40 \%$ of patients owing to tumor biological heterogeneity, host microenvironment complexity, drug resistance and other factors. Although certain mechanisms are involved in R-CHOP resistance, most patients with DLBCL present double-hit lymphoma, which involves double rearrangement of the $C-M Y C$ and $B C L-2$, or double-expression lymphoma, which shows high protein expression of both $C-M Y C$ and $B C L-$ 2 [13]. However, the present patient did not have double-hit lymphoma or double-expression lymphoma. This prompted us to consider other possible mechanisms for R-CHOP resistance. Scherer $e$ t al. have demonstrated that the mutation analysis of ctDNA can reveal the biological factors involved in the clinical outcome of lymphoma and help in the selection of individualized treatment [14]. Molecular monitoring of ctDNA has great potential and may be useful for monitoring the response to therapy or early relapse in various types of invasive B-cell lymphomas [15]. Through the regulation of downstream target genes, wild-type TP53 governs major defenses against tumor growth and promotes apoptosis, cellular DNA repair and cell cycle arrest [16]. On the other hand, mutant TP53 causes the loss of proliferation control, cell cycle dysregulation and genomic instability $[17,18]$. Following the progression of lymphoma in the current patient, a ctDNA test was performed, and it indicated the presence of a TP53 gene mutation that was clinically significant. This finding played a critical role in the subsequent decision-making process.

There is no consensus on the treatment of relapsed/refractory DLBCL with TP53 mutation. HDAC1 and HDAC2 can maintain the expression of mutant TP53, and small-molecule HDAC inhibitors can reduce the transcription and protein expression of mutant TP53 [19]. A Phase II study performed to evaluate the efficacy and safety of chidamide in Chinese patients with relapsed or refractory peripheral T-cell lymphoma has shown that chidamide acts as a novel benzamide-type subtype-selective HDAC inhibitor, with remarkable single-drug activity and controllable toxicity, and it provides a new therapeutic option for patients with peripheral T-cell lymphoma [20]. Moreover, clinical trials of chidamide for DLBCL are currently underway, and we look forward to encouraging results. HDAC inhibitors can overcome congenital and acquired rituximab resistance in patients with various types of B-cell lymphoma and augment the cytotoxic activity of rituximab by upregulating the expression of the CD20 
in lymphoma cells [5]. Chidamide, the new HDAC inhibitor available in China, would be an option for refractory DLBCL. Coleman et al. have used the PEP-C regimen (prednisone $20 \mathrm{mg}$, etoposide $50 \mathrm{mg} / \mathrm{m}^{2}$, procarbazine $50 \mathrm{mg} / \mathrm{m}^{2}$ and cyclophosphamide $50 \mathrm{mg} / \mathrm{m}^{2}$ ) to treat 75 cases of relapsed and refractory lymphomas in a clinical trial. The response rate for refractory DLBCL is $3 / 9$ and the time on treatment ranged from 3 weeks to 48 months (median 10 months). They have shown that the PEP-C regimen represents an active, tolerable treatment approach in patients with recurrent lymphoma [21]. This metronomic regimen might have been suitable for the present patient who was frail and refractory to current therapy and who needed control of accelerating lymphoma growth in a placid slope. Additionally, chidamide can induce mutant TP53 mRNA and protein drop in a time-dependent manner.

Thalidomide and lenalidomide are potent antineoplastic agents for myeloma as well as lymphoma. A Phase II clinical trial comparing thalidomide plus $\mathrm{CHOP}$ with $\mathrm{CHOP}$ alone has shown that the combination involving thalidomide significantly increased the complete response (CR) rate, particularly in patients with BCL-2-positive and BCL-6-negative DLBCL [22]. A case study has shown that two out of three relapsed elderly patients with DLBCL achieved complete remission using the combination of thalidomide $100 \mathrm{mg}$ and corticosteroids [23]. Two Phase II trials have shown that the addition of lenalidomide to R-CHOP appeared to mitigate the negative impact of the non-germinal center B-cell (GCB) phenotype on DLBCL outcome [24,25]. There are limited data on the effect of lenalidomide in relapsed or refractory DLBCL. Wang has reported the response rate of R2 regimen (rituximab plus lenalidomide) in relapsed DLBCL was about $28 \%$ with median 10.2 months OS [26]. The present patient experienced an allergic reaction on administration of lenalidomide; therefore, treatment was shifted to thalidomide.

\section{Conclusion}

Based on the efficacy and tolerability of the metronomic regimen, the possible mechanisms to overcome TP53 mutation through HDAC inhibitors, and in vitro experiment results, we believe that chidamide is a novel agent for treating patients with refractory DLBCL having TP53 mutation. We reported a progressive DLBCL with TP53 mutation that obtained sustained partial remission based on chidamide in combination with metronomic regimen. However, further clinical trials and experimental investigations are needed.

\section{Summary points}

- Diffuse large B-cell lymphoma (DLBCL) with TP53 mutation is often resistant to rituximab-based immunochemotherapy (R-CHOP) and has a poor prognosis.

- We report a case of a patient with R-CHOP-resistant senile DLBCL having TP53 mutation who showed sustained remission following treatment using a chidamide-based chemotherapy regimen.

- Furthermore, in vitro experiments demonstrated that chidamide has a greater lethal effect on DLBCL cell lines with mutant TP53 than on those with wild-type TP53. Moreover, chidamide has a therapeutic effect on DLBCL and may act by inhibiting the transcription and translation of mutated TP53 and upregulating the surface expression of the CD20 antigen in lymphoma cells.

- The chidamide-based chemotherapy regimen may be a new treatment option in patients with progressive DLBCL having TP53 mutation.

Financial \& competing interests disclosure

The research funding comes from National Natural Science Foundation of China 81470327. The authors have no other relevant affiliations or financial involvement with any organization or entity with a financial interest in or financial conflict with the subject matter or materials discussed in the manuscript apart from those disclosed.

No writing assistance was utilized in the production of this manuscript.

Ethical disclosure

The authors state that they have obtained appropriate institutional review board approval or have followed the principles outlined in the Declaration of Helsinki for all human or in vitro experimental investigations. In addition, for investigations involving human subjects, informed consent has been obtained from the participants involved. The authors state that they have obtained verbal and written informed consent from the patient/patients for the inclusion of their medical and treatment history within this case report. 


\section{Author contributions}

Q Li wrote the manuscript, Q Li and J Huang processed the in vitro experiment, Y Ou and Y Li collected the clinical data and did the follow-up. Y Wu designed the study and directed the project.

\section{Open access}

This work is licensed under the Attribution-NonCommercial-NoDerivatives 4.0 Unported License. To view a copy of this license, visit http://creativecommons.org/licenses/by-nc-nd/4.0/

\section{References}

Papers of special note have been highlighted as: $\bullet$ of interest

1 Kurtz DM, Scherer F, Jin MC et al. Circulating tumor DNA measurements as early outcome predictors in diffuse large B-cell lymphoma. J. Clin. Oncol. 36(28), 2845-2853 (2018).

2 Spina V, Bruscaggin A, Cuccaro A et al. Circulating tumor DNA reveals genetics, clonal evolution, and residual disease in classical Hodgkin lymphoma. Blood 131(22), 2413-2425 (2018).

3 Houldsworth J, Petlakh M, Olshen AB, Chaganti RS. Pathway activation in large B-cell non-Hodgkin lymphoma cell lines by doxorubicin reveals prognostic markers of in vivo response. Leuk. Lymphoma 49(11), 2170-2180 (2008).

4 Fernandez-Rodriguez C, Salar A, Navarro A et al. Anti-tumor activity of the combination of bendamustine with vorinostat in diffuse large B-cell lymphoma cells. Leuk. Lymphoma 57(3), 692-699 (2016).

5 Shimizu R, Kikuchi J, Wada T, Ozawa K, Kano Y, Furukawa Y. HDAC inhibitors augment cytotoxic activity of rituximab by upregulating CD20 expression on lymphoma cells. Leukemia 24(10), 1760-1768 (2010).

- HDAC inhibitors can overcome congenital and acquired rituximab resistance.

6 Siegel R, Naishadham D, Jemal A. Cancer statistics, 2013. CA Cancer J. Clin. 63(1), 11-30 (2013).

7 Pasqualucci L, Trifonov V, Fabbri G et al. Analysis of the coding genome of diffuse large B-cell lymphoma. Nat. Genet. 43(9), 830-837 (2011).

8 Lohr JG, Stojanov P, Lawrence MS et al. Discovery and prioritization of somatic mutations in diffuse large B-cell lymphoma (DLBCL) by whole-exome sequencing. Proc. Natl Acad. Sci. USA 109(10), 3879-3884 (2012).

9 Zenz T, Kreuz M, Fuge M et al. TP53 mutation and survival in aggressive B cell lymphoma. Int. J. Cancer 141(7), 1381-1388 (2017).

- $\quad$ TP53 mutations affect the prognosis of patients with diffuse large B-cell lymphoma (DLBCL).

10 Leroy $\mathrm{K}$, Haioun C, Lepage E et al. p53 gene mutations are associated with poor survival in low and low-intermediate risk diffuse large B-cell lymphomas. Ann. Oncol. 13(7), 1108-1115 (2002).

11 Ichikawa A, Kinoshita T, Watanabe T et al. Mutations of the p53 gene as a prognostic factor in aggressive B-cell lymphoma. N. Engl. J. Med. 337(8), 529-534 (1997).

12 Coiffier B, Thieblemont C, Van Den Neste E et al. Long-term outcome of patients in the LNH-98.5 trial, the first randomized study comparing rituximab-CHOP to standard CHOP chemotherapy in DLBCL patients: a study by the Groupe d'Etudes des Lymphomes de l'Adulte. Blood 116(12), 2040-2045 (2010).

13 Sarkozy C, Traverse-Glehen A, Coiffier B. Double-hit and double-protein-expression lymphomas: aggressive and refractory lymphomas. Lancet Oncol. 16(15), e555-e567 (2015).

14 Scherer F, Kurtz DM, Newman AM et al. Distinct biological subtypes and patterns of genome evolution in lymphoma revealed by circulating tumor DNA. Sci. Transl. Med. 8(364), 364 ra155 (2016).

15 Kwok M, Wu SP, Mo C, Summers T, Roschewski M. Circulating tumor DNA to monitor therapy for aggressive B-cell lymphomas. Curr. Treat. Options Oncol. 17(9), 47 (2016).

16 Whibley C, Pharoah PDP, Hollstein M. p53 polymorphisms: cancer implications. Nat. Rev. Cancer 9, 95 (2009).

17 Levine AJ, Hu W, Feng Z. The P53 pathway: what questions remain to be explored? Cell Death Differ. 13(6), 1027-1036 (2006).

18 Vogelstein B, Kinzler KW. Cancer genes and the pathways they control. Nat. Med. 10, 789 (2004).

19 Stojanovic N, Hassan Z, Wirth M et al. HDAC1 and HDAC2 integrate the expression of p53 mutants in pancreatic cancer. Oncogene 36(13), 1804-1815 (2017).

20 Shi Y, Dong M, Hong X et al. Results from a multicenter, open-label, pivotal Phase II study of chidamide in relapsed or refractory peripheral T-cell lymphoma. Ann. Oncol. 26(8), 1766-1771 (2015).

- Efficacy and safety of chidamide in patients with relapsed or refractory peripheral T-cell lymphoma in China.

21 Coleman M, Martin P, Ruan J et al. Prednisone, etoposide, procarbazine, and cyclophosphamide (PEP-C) oral combination chemotherapy regimen for recurring/refractory lymphoma: low-dose metronomic, multidrug therapy. Cancer 112(10), 2228-2232 (2008).

- Response rate and duration of PEP-C regimen in the treatment of relapsed and refractory lymphoma. 
22 Ji D, Li Q, Cao J et al. Thalidomide enhanced the efficacy of CHOP chemotherapy in the treatment of diffuse large B cell lymphoma: a Phase II study. Oncotarget 7(22), 33331-33339 (2016).

23 Wohner N, Varga G, Szloboda P et al. Thalidomide therapy in relapsed diffuse large B-cell lymphoma in elderly patients. Three cases. Orv. Hetil. 158(41), 1642-1648 (2017).

24 Nowakowski GS, Laplant B, Macon WR et al. Lenalidomide combined with R-CHOP overcomes negative prognostic impact of non-germinal center B-cell phenotype in newly diagnosed diffuse large B-cell lymphoma: a Phase II study. J. Clin. Oncol. 33(3), 251-257 (2015).

25 Vitolo U, Chiappella A, Franceschetti $S$ et al. Lenalidomide plus R-CHOP21 in elderly patients with untreated diffuse large B-cell lymphoma: results of the REAL07 open-label, multicentre, Phase II trial. Lancet Oncol. 15(7), 730-737 (2014).

- A Phase II trial of the effect of lenalidomide combined with R-CHOP on germinal center B-cell (GCB) and non-GCB DLBCL.

26 Wang M, Fowler N, Wagner-Bartak N et al. Oral lenalidomide with rituximab in relapsed or refractory diffuse large cell, follicular and transformed lymphoma: a Phase II clinical trial. Leukemia 27(9), 1902-1909 (2013).

- Therapeutic effect of $\mathbf{R} 2$ regimen (rituxmab plus lenalidomide) on relapsed DLBCL. 